\title{
Robust Adaptive Multiuser Detection for CDMA Frequency-Selective Fading Channels
}

\author{
Hongwei Zhou ${ }^{\dagger}$, Pei Xiao ${ }^{\dagger \dagger}$ \\ ${ }^{\dagger}$ Department of Civil Engineering, Imperial College London, SW7 2AZ, United Kingdom \\ E-mail: hongwei. zhoulimperial.ac.uk \\ ${ }^{\dagger \dagger}$ The Institute of Electronics, Communications and Information Technology \\ Queen's University Belfast, BT3 9DT, United Kingdom \\ E-mail: pei.xiao@ecit.qub.ac.uk
}

\begin{abstract}
Robust adaptive multiuser detection schemes are developed for direct-sequence code-division multiple-access (DSCDMA) multipath frequency-selective fading channels. Multiple access interference (MAI) and intersymbol interference (ISI) are presented in identical format in the expanded signal subspace, which provides convenience for symbol-by-symbol multiuser detection. The proposed multiuse detectors are designed in the expanded signal subspace, and subspace estimation and Kalman filtering algorithms are developed for their adaptive implementation. It is demonstrated by simulation that these adaptive detectors are robust against subspace estimation error and can effectively suppress both MAI and ISI and converge to the optimum SINR.
\end{abstract}

\section{INTRODUCTION}

Multiple access interference (MAI) has been a major impedance to achieve the promised advantages of code-division multiple-access (CDMA) technology in mobile communications. Intensive research interest has been attracted in recent years to develop multiuser detection (MUD) technologies to overcome MAI [1-5]. Multipath frequency-selective fading channels cause further practical concerns for broadband CDMA communications, which include the spreading waveform distortion and intersymbol interference (ISI). RAKE receiver [6] is a typical approach to tackle multipath problem, however, it is only optimum in single-user system. For multiuser systems, the RAKE structure can still be adopted but the matched filter bank is replaced by linear multiuser detector bank [7-9]. These schemes hold a common assumption that ISI is negligible. This paper aims at multiuser detection problem for multipath channels with arbitrarily long delay so that ISI is not negligible.

The development effort begins with deriving an analytical one-shot signal model. Instead to suppress the echo multipath components, an extended observation window and reformed user spreading codes are adopted so that all multipath components are used for the detection of one symbol. In this signal model, the ISI and MAI appear in distinct format and hence both can be suppressed by multiuser detection technology. Subspace technology is used in [3] to realize blind multiuser detection in additive white Gaussian noise (AWGN) channel. Wiener minimum mean-square-error (MMSE) detector is well known for being optimum in the MSE sense. It is proven in this paper that it is also an optimum SINR detector and lies in the signal subspace. This provides another angle to appreciate the MMSE detector. For a system with fixed configuration, there exists a SINR upper bound and it is expressed in closed form. This motivates us to design multiuser detector in the expanded signal subspace. If the channel and the autocorrelation matrix of the received signals are precisely known, then Wiener MMSE detector is readily available [10], however, in practical systems, accurate knowledge of the channel and autocorrelation matrix is hardly available. Such inaccuracy will seriously deteriorate the performance of the Wiener MMSE detector, as will be shown by simulation. Adaptive multiuser detection strategies are proposed in this paper. The subspace-based detector can be decomposed along all orthogonal directions in expanded signal subspace. Deflated batch processing method or the modified projection approximation subspace tracking (PAST) method [11] can be used to estimate the signal subspace, and Kalman filter can be used to estimate the coefficients along each subspace basis. These adaptive detectors are proven by simulation to be efficient in suppressing both MAI and ISI and they approach the optimum SINR performance in multipath channels.

\section{SySTEM MODEL}

Consider a synchronous $K$-user DS-CDMA system employing BPSK modulation to transmit signals through multipath channels. The symbol interval and chip interval are denoted by $T$ and $T_{c}$, respectively. The $k$ th user's spreading waveform is

$$
c_{k}(t)=\sum_{n=0}^{N-1} c_{k}(n) \psi\left(t-n T_{c}\right), t \in[0, T], k=1, \ldots, K,
$$

where $N=T / T_{c}$ is the spreading factor; $\left\{c_{k}(n)\right\}_{n=0}^{N-1}$ is the normalized signature code assigned to the $k$ th user; $\psi(t)$ is a normalized chip waveform defined in $\left[\begin{array}{ll}0 & T_{c}\end{array}\right]$. The multipath channel is modeled by a tapped delay line with tap spacing $1 / W$ ( $W$ is the bandwidth of the spread-spectrum signals) and tap coefficients $\left\{h_{k}(l)\right\}_{l=0}^{L-1}$, where $L$ is the number of resolvable paths for each user. We consider the case when $W=1 / T_{c}, L=\left\lceil T_{m} / T_{c}\right\rceil$, where $T_{m}$ is the multipath delay spread, and $\lceil x\rceil$ denotes the value obtained by rounding $x$ to the nearest integer which is greater or equal to $x$. Transmitting the signals through the multipath channel, the received signal due to the $k$ th user is given by

$$
y_{k}(t)=\sum_{i=-\infty}^{\infty} A_{k} b_{k}(i) \sum_{l=0}^{L-1} h_{k}(l) c_{k}(t-i T-l / W),
$$


where $A_{k}$ and $b_{k}(i)$ are the amplitude, the $i$ th transmitted information bit of the $k$ th user, respectively; $b_{k}(i) \in\{+1,-1\}$ follows identical independent distribution (i.i.d.). The total received signal is the superposition of the information-bearing data signals of $K$ users plus the additive white Gaussian noise (AWGN), i.e., $r(t)=\sum_{k=1}^{K} y_{k}(t)+v(t)$, where $v(t)$ is the zero-mean white Gaussian noise with variance $\sigma^{2}$. After chipmatched filtering followed by chip-rate sampling, the discretetime format of the received signal is given by

$$
r(j)=\sum_{k=1}^{K} y_{k}(j)+v(j),
$$

where

$$
\begin{aligned}
y_{k}(j) & =\sum_{i=-\infty}^{\infty} A_{k} b_{k}(i) s_{k}(j-i N) ; \\
s_{k}(n) & =\sum_{l=0}^{L-1} h_{k}(l) c_{k}(n-l) ; \\
v(j) & =\int_{j T_{c}}^{(j+1) T_{c}} v(t) \psi\left(t-j T_{c}\right) d t .
\end{aligned}
$$

On the transmitter side, the energy of each symbol is limited in duration $N T_{c}$. However, on the receiver side, the energy is spread over an extended interval $(N+L-1) T_{c}$ due to the channel convolution effect, which can be observed from (2). We aim to design a multiuser detector that detects the signals in a symbol-by-symbol fashion and employ a processing window of length $N+L-1$ to model the received signal in a vector form. Falling within the window are not only the information bits for the current time instant, but also those before and after the current time instant. The received signal vector thus contains ISI from the past as well as the future symbols. In order to describe it quantitatively, we define $P=\lceil(L-1) / N\rceil$. Then, $2 P+1$ symbols from the desired user are included in the processing window of length $N+L-1$, including the current symbol, $P$ symbols before and $P$ symbols after the current symbol. Taking into account of all the $\mathrm{K}$ users, there are $(2 P+1) K$ symbols involved in detecting one symbol of the desired user. The $(N+L-1)$-element received signal vector can be expressed as

$$
\mathbf{r}(i)=\sum_{k=1}^{K} \mathbf{y}_{k}(i)+\mathbf{v}(i)
$$

where

$$
\begin{aligned}
& \mathbf{y}_{k}(i)=A_{k} b_{k}(i) \mathbf{s}_{k}+\sum_{j=1}^{P} A_{k} b_{k}(i-j) \underline{\mathbf{s}_{k, j}} \\
& +\sum_{j=1}^{P} A_{k} b_{k}(i+j) \underline{\underline{\underline{\mathbf{s}_{k, j}}}} ; \\
& \mathbf{s}_{k}=\left[\begin{array}{lll}
s_{k}(0) & \ldots & s_{k}(N+L-2)
\end{array}\right]^{\mathcal{T}} ; \\
& \underline{\mathbf{s}_{k, j}}=\left[\begin{array}{llllll}
s_{k}(j N) & \ldots & s_{k}(N+L-2) & 0 & \ldots & 0
\end{array}\right]^{\mathcal{T}} ; \\
& \underline{\underline{\mathbf{s}_{k, j}}}=\left[\begin{array}{llllll}
0 & \ldots & 0 & s_{k}(0) & \ldots & s_{k}(N+L-2-j N)
\end{array}\right]^{\mathcal{T}} \text {; } \\
& \mathbf{v}(i)=\left[\begin{array}{lll}
v(i N) & \ldots & v(i N+N+L-2)
\end{array}\right]^{\mathcal{T}} .
\end{aligned}
$$

Define

$$
\begin{aligned}
& \underline{\mathbf{s}_{k}}=\left[\begin{array}{lll}
\underline{\mathbf{s}_{k, 1}} & \cdots & \underline{\mathbf{s}_{k, P}}
\end{array}\right] ; \quad \underline{\underline{\mathbf{s}_{k}}}=\left[\begin{array}{lll}
\underline{\underline{\mathbf{s}_{k, 1}}} & \cdots & \underline{\underline{\mathbf{s}_{k, P}}}
\end{array}\right] ; \\
& \mathbf{S}=\left[\begin{array}{llllllllll}
\mathbf{s}_{1} & \underline{\mathbf{s}_{1}} & \underline{\underline{\mathbf{s}_{1}}} & \mathbf{s}_{2} & \underline{\mathbf{s}_{2}} & \underline{\underline{\mathbf{s}_{2}}} & \ldots & \mathbf{s}_{K} & \underline{\mathbf{s}_{K}} & \underline{\underline{\mathbf{s}_{K}}}
\end{array}\right]^{\mathcal{T}} ; \\
& \mathbf{A}=\operatorname{diag}\{\underbrace{A_{1} \ldots A_{1}}_{2 P+1} \underbrace{A_{2} \ldots A_{2}}_{2 P+1} \cdots \underbrace{A_{k} \ldots A_{k}}_{2 P+1}\} ; \\
& \mathbf{b}_{k}(i)=\left[\begin{array}{llllll}
b_{k}(i) & \ldots & b_{k}(i-P) & b_{k}(i+1) & \ldots & b_{k}(i+P)
\end{array}\right] ; \\
& \mathbf{b}(i)=\left[\begin{array}{lll}
\mathbf{b}_{1}(i) & \ldots & \mathbf{b}_{K}(i)
\end{array}\right]^{\mathcal{T}},
\end{aligned}
$$

then (4) can be written in a compact form as

$$
\mathbf{r}(i)=\mathbf{S A} \mathbf{b}(i)+\mathbf{v}(i) .
$$

In (5), $b_{k}(i), b_{k}(i-j), b_{k}(i+j)$ are all from the $k$ th user. However, they can be regarded as statistically independent signals from different users which include the $k$ th user with modified signature codes $\mathbf{s}_{k}$, and $(2 P+1)$ virtual users with signature codes $\mathbf{s}_{k, j}$ and $\mathbf{s}_{k, j}$. Performing eigendecomposition of the autocorrelation matrix $\mathbf{R}=\mathrm{E}\left[\mathbf{r r}^{\mathcal{T}}\right]$ yields

$\mathbf{R}=\left[\begin{array}{ll}\mathbf{U}_{s} & \mathbf{U}_{n}\end{array}\right]\left[\begin{array}{cc}\Lambda_{s}+\sigma^{2} \mathbf{I}_{s} & \mathbf{0} \\ \mathbf{0} & \sigma^{2} \mathbf{I}_{n}\end{array}\right]\left[\begin{array}{c}\mathbf{U}_{s}^{\mathcal{T}} \\ \mathbf{U}_{n}^{\mathcal{T}}\end{array}\right]=\mathbf{U}_{s} \Lambda_{s} \mathbf{U}_{s}^{\mathcal{T}}+\sigma^{2} \mathbf{I}$,

where $\Lambda_{s}$ is a diagonal matrix whose diagonal elements are positive. $\Lambda_{s}+\sigma^{2} \mathbf{I}_{s}$ contains the most significant eigenvalues of $\mathbf{R}$ that are not equal to $\sigma^{2}$ and $\mathbf{U}_{s}$ contains the corresponding orthonormal eigenvectors; $\sigma^{2} \mathbf{I}_{n}$ contains eigenvalues that are equal to $\sigma^{2}$ and $\mathbf{U}_{n}$ contains the corresponding orthonormal eigenvectors. $\mathbf{I}_{s}, \mathbf{I}_{n}$ and $\mathbf{I}$ are identity matrices. The columns of $\mathbf{U}_{s}$ span the signal subspace and the columns of $\mathbf{U}_{n}$ span the noise subspace. It is easy to understand that $\mathbf{S}$ and $\mathbf{U}_{s}$ have the same rank and span the same subspace.

The signal model and the detectors in this paper are derived for synchronous multipath channels, however, if the user delays are known or estimated, the asynchronous multipath channels can be directly modelled in similar format as the synchronous channels, and hence the developed adaptive multiuser detectors can also be applied directly.

\section{Optimal MULTIUSER DETECTORS}

Assume that user 1 is the interested user. Any linear multiuser detector for user 1 can be expressed as

$$
\mathbf{d}_{1}=\mathbf{U}_{s} \boldsymbol{\alpha}+\mathbf{U}_{n} \boldsymbol{\beta}=\mathbf{d}_{1 s}+\mathbf{d}_{1 n},
$$

where $\boldsymbol{\alpha}$ is a $(2 P+1) K \times 1$ weight vector and $\boldsymbol{\beta}$ is a $[N+$ $L-1-(2 P+1) K] \times 1$ weight vector, $\mathbf{d}_{1}=\mathbf{U}_{s} \boldsymbol{\alpha}$ is the projection of the detector in the signal subspace and $\mathbf{d}_{1 n}=$ $\mathbf{U}_{n} \boldsymbol{\beta}$ is the projection in the noise subspace. We know that the linear detector with optimum SINR performance must exist in the signal subspace.

Proposition 1: MMSE detector is an optimum SINR detector and lies in the signal subspace.

Proof: Assume that $\mathbf{d}_{1}$ is an optimum detector with maxi2 mum SINR. Let $\alpha=\frac{1}{\mathbf{d}_{1}^{1} \mathbf{s}_{1}}$. If $\mathbf{d}_{1}$ is the optimum detector with 
maximum SINR, then $\alpha \mathbf{d}_{1}$ is also an optimum detector and has the same maximum SINR

$$
\begin{aligned}
S I N R\left(\alpha \mathbf{d}_{1}\right) & =\frac{\mathrm{E}\left[\left(\alpha \mathbf{d}_{1}\right)^{\mathcal{T}} A_{1} b_{1}(i) \mathbf{s}_{1}\right]^{2}}{\mathrm{E}\left[\left(\alpha \mathbf{d}_{1}\right)^{\mathcal{T}} \mathbf{r}-A_{1} b_{1}(i) \mathbf{s}_{1}\right]^{2}} \\
& =\frac{\mathrm{E}\left[A_{1} b_{1}(i) \alpha \mathbf{d}_{1}^{\mathcal{T}} \mathbf{s}_{1}\right]^{2}}{\mathrm{E}\left[\alpha \mathbf{d}_{1}^{\mathcal{T}} \mathbf{r}-A_{1} b_{1}(i) \alpha \mathbf{d}_{1}^{\mathcal{T}} \mathbf{s}_{1}\right]^{2}} \\
& =\frac{A_{1}^{2}}{\mathrm{E}\left[\alpha \mathbf{d}_{1}^{\mathcal{T}} \mathbf{r}-A_{1} b_{1}(i)\right]^{2}}
\end{aligned}
$$

Define

$$
\mathbf{d}_{M M S E}=\alpha \mathbf{d}_{1},
$$

it is straightforward to show that

$$
\begin{aligned}
\mathbf{d}_{M M S E} & =\arg \max _{\mathbf{d}} \frac{A_{1}^{2}}{\mathrm{E}\left[\mathbf{d}^{\mathcal{T}} \mathbf{r}-A_{1} b_{1}(i)\right]^{2}} \\
& =\arg \min _{\mathbf{d}} \mathrm{E}\left[\mathbf{d}^{\mathcal{T}} \mathbf{r}-A_{1} b_{1}(i)\right]^{2},
\end{aligned}
$$

for which an implicit constraint is

$$
\mathbf{d}^{\mathcal{T}} \mathbf{s}_{1}=1 \text {. }
$$

The solution to (10) is the MMSE detector. Note that, according to (9), this MMSE detector also achieves the maximum SINR. Therefore, this MMSE detector is optimum in terms of both SINR and mean square error, and the MMSE detector is in the signal subspace.

Discussion:

1. According to (8) and (10), for any optimum SINR detector $\mathbf{d}_{1}, \mathbf{d}_{1} /\left(\mathbf{d}_{1}^{\mathcal{T}} \mathbf{s}_{1}\right)$ is the MMSE detector.

2. The solution to the constrained minimization problem in (10) and (11) is

$$
\mathbf{d}_{C M M S E}=\frac{\mathbf{R}^{-1} \mathbf{s}_{1}}{\mathbf{s}_{1}^{\mathcal{T}} \mathbf{R}^{-1} \mathbf{s}_{1}},
$$

where $\mathbf{d}_{C M M S E}$ means constrained MMSE detector.

3. Without the constraint in (11), solving (10) leads to the Wiener solution

$$
\mathbf{d}_{W M M S E}=A_{1}^{2} \mathbf{R}^{-1} \mathbf{s}_{1} \text {. }
$$

Comparing (12) and (13), it can be seen that the Wiener detector is scalar times of the constrained MMSE detector. According to Proposition 1, they should achieve the same maximum output SINR which is

$$
\begin{aligned}
& \operatorname{SINR}\left(\mathbf{d}_{C M M S E}\right)=\operatorname{SINR}\left(\mathbf{d}_{W M M S E}\right) \\
& =\frac{A_{1}^{2}}{1 /\left(\mathbf{s}_{1}^{\mathcal{T}} \mathbf{R}^{-1} \mathbf{s}_{1}\right)-A_{1}^{2}}
\end{aligned}
$$

But $\mathbf{d}_{C M M S E}$ and $\mathbf{d}_{W M M S E}$ have different mean square errors, which are given by

$$
\begin{aligned}
M S E\left(\mathbf{d}_{C M M S E}\right) & =\frac{1}{\mathbf{s}_{1}^{\mathcal{T}} \mathbf{R}^{-1} \mathbf{s}_{1}}-A_{1}^{2} \\
M S E\left(\mathbf{d}_{W M M S E}\right) & =A_{1}^{2}-A_{1}^{4} \mathbf{s}_{1}^{\mathcal{T}} \mathbf{R}^{-1} \mathbf{s}_{1}
\end{aligned}
$$

Theoretical value of the autocorrelation matrix can be computed by

$$
\mathbf{R}=\mathbf{S A}^{2} \mathbf{S}^{\mathcal{T}}+\sigma^{2} \mathbf{I}_{(N+L-1) \times(N+L-1)},
$$

where

$$
\mathbf{A}_{(2 P+1) K \times(2 P+1) K}^{2}=\operatorname{diag}\left\{A_{1}^{2}, \ldots, A_{1}^{2}, \ldots, A_{K}^{2}, \ldots, A_{K}^{2}\right\} .
$$

IV. Adaptive Multiuser Detectors for Multipath

\section{Channels}

From (12) and (13), it can be seen that to calculate the MMSE detector, we first need know the autocorrelation matrix and then do matrix inversion. In practice, the autocorrelation matrix can be estimated by time average:

$$
\mathbf{R}=\mathrm{E}\left[\mathbf{r r}^{\mathcal{T}}\right] \approx \frac{1}{M} \sum_{i=1}^{M} \mathbf{r}(i) \mathbf{r}(i)^{\mathcal{T}}
$$

The larger $M$, the more precise estimation. However, a larger $M$ means longer delay, and matrix inversion involves higher computation complexity especially when $N+L-1$ is large. The performance of the direct MMSE detector implementation is affected by the estimation error to a great extend as will be shown by simulation. The matrix inversion operation is also expensive in terms of computational complexity. We propose adaptive multiuser detection schemes for multipath channels. As in (7), the desired detector can be decomposed in the signal subspace $\mathbb{R}^{(N+L-1) \times(2 P+1) K}$

$$
\mathbf{d}_{1}=\mathbf{U}_{s} \boldsymbol{\alpha}=\mathbf{s}_{1}+\mathbf{U}_{s, \perp} \mathbf{w}
$$

where $\mathbf{U}_{s, \perp}$ is a $(N+L-1) \times[(2 P+1) K-1]$ matrix and its column vectors are all in the expanded signal subspace and are orthogonal with $\mathbf{s}_{1} ; \mathbf{w}$ is a $[(2 P+1) K-1] \times 1$ weight vector. To pursue a MMSE solution, the constraint in (11) is applied. This constraint can be integrated into the expression of $\mathbf{d}_{1}$ as follows

$$
\mathbf{d}_{1}=\frac{\mathbf{s}_{1}}{\left\|\mathbf{s}_{1}\right\|^{2}}+\mathbf{U}_{s, \perp} \mathbf{w}
$$

It can be easily shown that $\mathbf{d}_{1}^{\mathcal{T}} \mathbf{s}_{1}=1$. Now the tasks are to estimate the signal subspace basis $\mathbf{U}_{s, \perp}$ and the coefficient vector $\mathbf{w}$. We process estimation of $\mathbf{w}$ using Kalman filter first and then present two methods to estimate $\mathbf{U}_{s, \perp}$.

\section{A. Estimate w by Kalman Filter}

Assume that $\mathbf{U}_{s, \perp}$ has been estimated. For stationary system, the state space model is given by

$$
\begin{aligned}
& \mathbf{w}(i)=\mathbf{w}(i-1) \quad \text { state transition equation } \\
& y(i)=\mathbf{H}^{\mathcal{T}}(i) \mathbf{w}(i)+e(i) \quad \text { measurement equation }
\end{aligned}
$$

where

$$
\begin{aligned}
& y(i)=\frac{\mathbf{s}_{1}^{\mathcal{T}} \mathbf{r}(i)}{\left\|\mathbf{s}_{1}\right\|^{2}} \\
& \mathbf{H}^{\mathcal{T}}(i)=-\mathbf{r}(i)^{\mathcal{T}} \mathbf{U}_{s, \perp} \\
& e(i)=\mathbf{d}_{1}^{\mathcal{T}} \mathbf{r}(i)=\frac{\mathbf{s}_{1}^{\mathcal{T}} \mathbf{r}(i)}{\left\|\mathbf{s}_{1}\right\|^{2}}+\mathbf{r}^{\mathcal{T}}(i) \mathbf{U}_{s, \perp} \mathbf{w}(i),
\end{aligned}
$$

where $e(i)$ is the measurement noise. Its mean and variance are

$$
\begin{aligned}
& \mu=\mathrm{E}[e(i)]=\mathrm{E}\left[\mathbf{d}_{1}^{\mathcal{T}}(i) \mathbf{r}(i)\right]=\mathbf{d}_{1}^{\mathcal{T}} \mathrm{E}[\mathbf{r}(i)]=0 \\
& \varphi=\operatorname{cov}[e(i)]=\mathrm{E}\left\{[e(i)-\mu]^{2}\right\}=\mathrm{E}\left[e^{2}(i)\right]=\mathrm{E}\left[\mathbf{d}_{1}^{\mathcal{T}} \mathbf{r}(i)\right]^{2}
\end{aligned}
$$

The mean square error of the detector is

$$
\varepsilon=\mathrm{E}\left[\mathbf{d}_{1}^{\mathcal{T}} \mathbf{r}(i)-A_{1} b_{1}(i)\right]^{2}=\mathrm{E}\left[\mathbf{d}_{1}^{\mathcal{T}} \mathbf{r}(i)\right]^{2}-A_{1}^{2} .
$$


Hence

$$
\varphi=A_{1}^{2}+\varepsilon
$$

When the detctor converges to the MMSE detector

$$
\varphi_{\min }=A_{1}^{2}+\varepsilon_{\min } .
$$

In high $\mathrm{SNR}$ scenario, the minimum mean square error $\varepsilon_{\min }=1 / \mathbf{s}_{1}^{\mathcal{T}} \mathbf{R}^{-1} \mathbf{s}_{1}-A_{1}^{2} \approx 0$, therefore $\varphi_{\min } \approx A_{1}^{2}$.

\section{B. Estimate $\mathbf{U}_{s, \perp}$ by Batch Processing Method}

Because $\mathbf{U}_{s, \perp}$ contains only subspace basis that are orthogonal with $\mathbf{s}_{1}$, the received signal can be deflated by projecting the received signal onto $\mathbf{s}_{1}$ and then subtracting the projection from the received signal as follows

$$
\mathbf{r}_{\perp}(i)=\mathbf{r}(i)-\mathbf{r}(i)^{\mathcal{T}} \mathbf{s}_{1} \frac{\mathbf{s}_{1}}{\left\|\mathbf{s}_{1}\right\|^{2}}
$$

It is easy to verify that $\mathbf{r}_{\perp}(i)$ and $\mathbf{s}_{1}$ are orthogonal, therefore it can be expected that the subspace basis extracted from $\mathbf{r}_{\perp}(i)$ will automatically be orthogonal with $\mathbf{s}_{1}$. The autocorrelation matrix is estimated by time average as follows

$$
\mathbf{R}_{\perp}=\frac{1}{M} \sum_{i=1}^{M} \mathbf{r}_{\perp}(i) \mathbf{r}_{\perp}(i)^{\mathcal{T}}
$$

By applying eigendecomposition to $\mathbf{R}_{\perp}$, the $(N+L-1) \times$ $[(2 P+1) K-1]$ eigenvector matrix $\mathbf{U}_{s, \perp}$ is obtained. Here we also have the problem of inaccurate estimation of $\mathbf{R}_{\perp}$, but the simulation will show that the Kalman filter-based multiuser detector is robust against the subspace estimation error. Hence a large batch $M$ is not necessary.

\section{Estimate $\mathbf{U}_{s, \perp}$ by Windowed PAST Algorithm}

The batch processing-based subspace estimation methods approaches have time delay problem in signal detection, and the required batch eigenvalue decomposition (ED) or singular value decomposition (SVD) operation has computational complexity $O\left((N+L-1)^{3}\right)$. Projection approximation subspace tracking (PAST) is a different approach which relies on a novel interpretation that the signal subspace is the solution to an unconstrained minimisation problem. This lends convenience for adaptive implementation. PAST algorithm guarantees global convergence and has linear complexity. To integrate the PAST subspace tracking algorithm into the proposed multiuser detector, three modifications are made to it. First, the received signal is pre-processed by deflation operation as in (15) for the same reason as stated earlier. Secondly, the recursive least square (RLS) version of PAST does not guarantee that the output eigenvectors are orthonormal, hence one explicit orthonormalization step is introduced. Thirdly, tracking window is imposed to the PAST algorithm, that is, the subspace tracking process only lasts for limited time duration. Intuitive belief is that the more data used for subspace tracking, the better accuracy will be achieved and the better performance the detector will have. However, because Kalman filtering tracking and the subspace tracking are two separate dynamic processes, and the Kalman filtering tracking is based on the subspace tracking output, frequently changing the subspace basis affects the convergence of the Kalman filter. The windowing scheme enables initial subspace tracking and then stops it. This avoids the error propagation in the Kalman filter tracking and allows it to converge. Kalman filter has strong tracking ability based on not-exactlyestimated subspace basis. This windowing scheme also reduces the computational complexity. This algorithm is summarized in Table 1.

Table 1: Multiuser detector for multipath channels using the windowed PAST subspace tracking and Kalman filter-based weight tracking algorithms ( $\beta$ is the forgetting factor)

1. Initialize subspace parameters $\mathbf{G}(0)$ and $\mathbf{U}_{s, \perp}(0)$;

Initialize Kalman filter parameter $\mathbf{P}(0)$ and weight $\mathbf{w}(0)$. 2. / $* \mathbf{w}$ Parameter tracking and signal detection $*$ / FOR $i=1,2, \ldots$

/* PAST-based subspace tracking */

IF $i<M \quad / *$ window width is $M * /$

$$
\begin{aligned}
\mathbf{r}_{\perp}(i) & =\mathbf{r}(i)-\mathbf{r}(i)^{\mathcal{T}} \mathbf{s}_{1} \mathbf{s}_{1} /\left\|\mathbf{s}_{1}\right\|^{2} \\
\mathbf{y}(i) & =\mathbf{U}_{s, \perp}^{\mathcal{T}}(i-1) \mathbf{r}_{\perp}(i) \\
\mathbf{h}(i) & =\mathbf{G}(i-1) \mathbf{y}(i) \\
\mathbf{g}(i) & =\mathbf{h}(i) /\left[\beta+\mathbf{y}(i)^{\mathcal{T}} \mathbf{h}(i)\right] \\
\mathbf{G}(i) & =1 / \beta\left[\mathbf{G}(i-1)-\mathbf{g}(i) \mathbf{h}(i)^{\mathcal{T}}\right] \\
\mathbf{e}(i) & =\mathbf{r}_{\perp}(i)-\mathbf{U}_{s}(i-1) \mathbf{y}(i) \\
\mathbf{U}_{s, \perp}(i) & =\mathbf{U}_{s, \perp}(i-1)+\mathbf{e}(i) \mathbf{g}(i)^{T}
\end{aligned}
$$

Orthornormalize $\left[\mathbf{s}_{1} \quad \mathbf{U}_{s, \perp}(i)\right]$.

END

/* Kalman filter tracking */

$$
\begin{aligned}
y(i) & =\mathbf{s}_{1}^{\mathcal{T}} \mathbf{r}(i) /\left\|\mathbf{s}_{1}\right\|^{2} \\
\mathbf{H}^{\mathcal{T}}(i) & =-\mathbf{r}(i)^{\mathcal{T}} \mathbf{U}_{s, \perp} \\
\mathbf{k}(i) & =\mathbf{P}(i-1) \mathbf{H}(i)\left[\mathbf{H}(i)^{\mathcal{T}} \mathbf{P}(i-1) \mathbf{H}(i)+\varphi_{\min }\right]^{-1} \\
\mathbf{P}(i) & =\left[\mathbf{I}-\mathbf{k}(i) \mathbf{H}(i)^{\mathcal{T}}\right] \mathbf{P}(i-1) \\
\mathbf{w}(i) & =\mathbf{w}(i-1)+\mathbf{k}(i)\left[y(i)-\mathbf{H}(i)^{\mathcal{T}} \mathbf{w}(i-1)\right]
\end{aligned}
$$

/*signal detection*/

$$
\begin{aligned}
\mathbf{d}_{1}(i) & =\mathbf{s}_{1} /\left\|\mathbf{s}_{1}\right\|^{2}+\mathbf{U}_{s, \perp} \mathbf{w}(i) \\
\hat{b}(i) & =\operatorname{sgn}\left\{\mathbf{d}_{1}(i)^{\mathcal{T}} \mathbf{r}(i)\right\}
\end{aligned}
$$

END

\section{Numerical Results}

In the simulated system, there are 10 users $(K=10)$. User 1 is assumed to be the desired one and has fixed SNR 20dB (compared with the noise). Compared with user 1, users 2 to 6 are $10 \mathrm{~dB}$ stronger, users 7 to 9 are $20 \mathrm{~dB}$ stronger, and users 10 is $30 \mathrm{~dB}$ stronger, respectively. The spreading codes are randomly generated and normalized, and the spreading gain is $N=31$. The multipath channel coefficients of each user are randomly generated and normalized and have exponential decay profile. All presented results are the average of 1000 Monte Carlo simulations.

Example 1: This example studies the effect of the batch length in the batch processing-based subspace estimation on 
the detectors. The expanded subspace and Kalman filter-based multiuser detector is compared with the Wiener filter in (13). Batch processing method is used to estimate $\mathbf{U}_{s, \perp}$ for the former and is used to estimate the correlation matrix $\mathbf{R}$ for the latter. Figure 1(a) shows the results when the multipath channel spread is 25 , while Figure 1(b) corresponds to the channel spread 56 (longer than one symbol duration). Batches of 100 , 500 and 10000 symbols are tested for both detectors. It is seen that the Wiener filter is very sensitive to batch length. More symbols are used, more accurate the estimated correlation matrix is. When batch length is 10000 , the SINR performance nearly approaches the SINR upper bound of any linear multiuser detector. The SINR upper bound is calculated according to (14). Because the SINR bound is specific to the system setting, and the system settings (channel coefficients and user spreading codes) vary in each Monte Carlo run, hence the plotted SINR bound is the average of all Monte Carlo simulations. It is also seen that the expanded subspace and Kalman filterbased detector is almost not affected by the batch length, in other words, it is robust against the subspace estimation error.

Example 2: this example examines the effect of the window length on the expanded subspace and Kalman filter-based multiuser detector in Table 1. In Figure 2, windows of 50, 150 and 500 symbols are examined for subspace tracking. The batch processing-based adaptive multiuser detector inevitably has delay, but it only need do Kalman filtering tracking once the signal detection phase begins. The windowed PAST-based adaptive multiuser detector in Table 1 has advantage in terms of no delay, but during the window interval, it needs carry out both subspace tracking and Kalman filter tracking in each signal detecting iteration. Shorter window demands computation for shorter period. The simulation results in Figure 2 show that windows of 50 and 500 achieve similar performance. It reflects the robustness of the adaptive detector.

\section{CONCLUSIONS}

Multiple access interference and multipath frequencyselecting fading are the two impedances in high-speed CDMA communications. Two adaptive multiuser detectors are developed in the expanded signal subspace by using subspace estimation and Kalman filtering estimation algorithms. They effectively suppress both MAI and ISI simultaneously and approach the optimum SINR performance bound, and are much more robust against subspace estimation error than the Wiener MMSE detector.

\section{REFERENCES}

[1] S. Verdu. Multiuser Detection. Cambridge University Press, 2001.

[2] M.L. Honig, U. Madhow and S. Verdu, "Blind adaptive multiuser detection", IEEE Trans. Information Theory, vol. 41, no. 4, pp. 944-960, July 1995.

[3] X. Wang and H.V. Poor "Blind Multiuser Detection: A Subspace Approach," IEEE Trans. on Information Theory, vol. 44, no. 2, pp. 677-690, March 1998.

[4] X. Zhang and W. Wei, "Blind adaptive multiuser detection based on Kalman filtering," IEEE Trans. on Signal Processing, vol. 50, no. 1, pp. 87-94, Jan. 2002.

[5] H. Zhou, W.L. Woo, and B.S. Sharif, "Subspace-based blind adaptive multiuser detection using Kalman filter," IEE Proc. Commun., vol. 152, no. 3, pp. 302-310, June 2005.

[6] J. G. Proakis. Digital Communications. Boston: McGraw-Hill, 4th edition, 2001.

[7] X. Wang and H.V. Poor, "Blind adaptive multiuser detection in multipath CDMA channels based on subspace tracking," IEEE Trans. Signal Processing, vol. 46, no. 11, pp. 3030-3044, 1998.
[8] H. Liu and K. Li, "A decorrelating RAKE receiver for CDMA communications over frequency- selective fading channels," IEEE Trans. Commun., vol. 47, no. 7, pp. 1036-1045, 1999.

[9] T. Liu, "Linearly constrained minimum variance filters for blind multiuser detection," IEEE Trans. Commun., vol. 51, no. 10, pp. 1649-1652, 2003.

[10] S. Haykin, Adaptive Filter Theory. NJ: Prentice-Hall, 4th edition, 2002.

[11] B. Yang, "Projection Approximation Subspace Tracking," IEEE Trans. Signal Processing, vol. 43, pp. 95-107, 1995. 


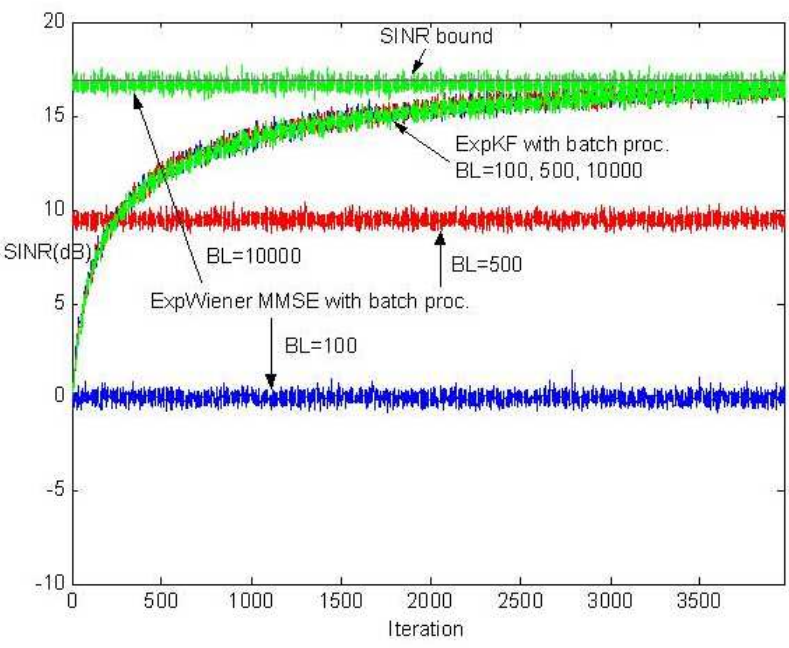

(a) channel spread $=25$.

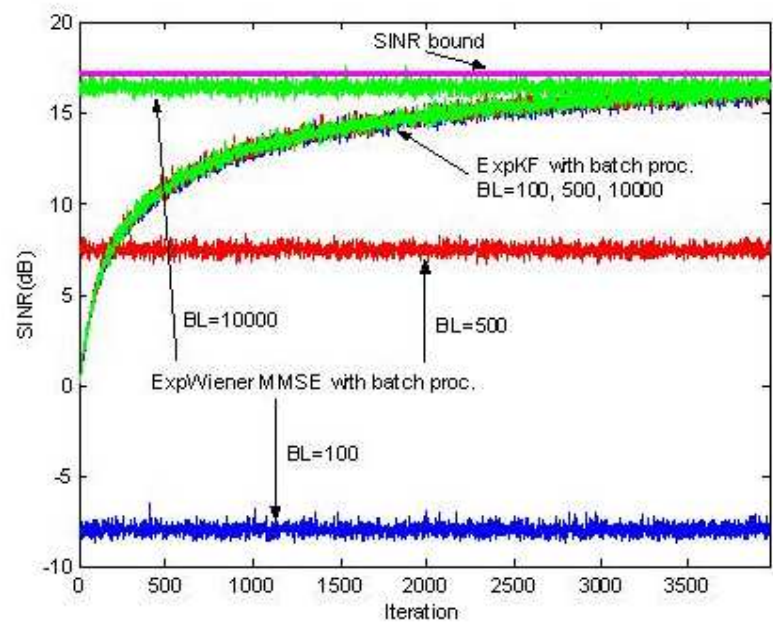

(b) channel spread $=56$.

Fig. 1. $(K=10, N=31, \mathrm{SNR}=20 \mathrm{~dB})$ SINR performance of the expanded subspace Kalman filter-based adaptive detector and the Wiener MMSE detector in multipath channels. Subspace and autocorrelation matrix are estimated by batch processing.

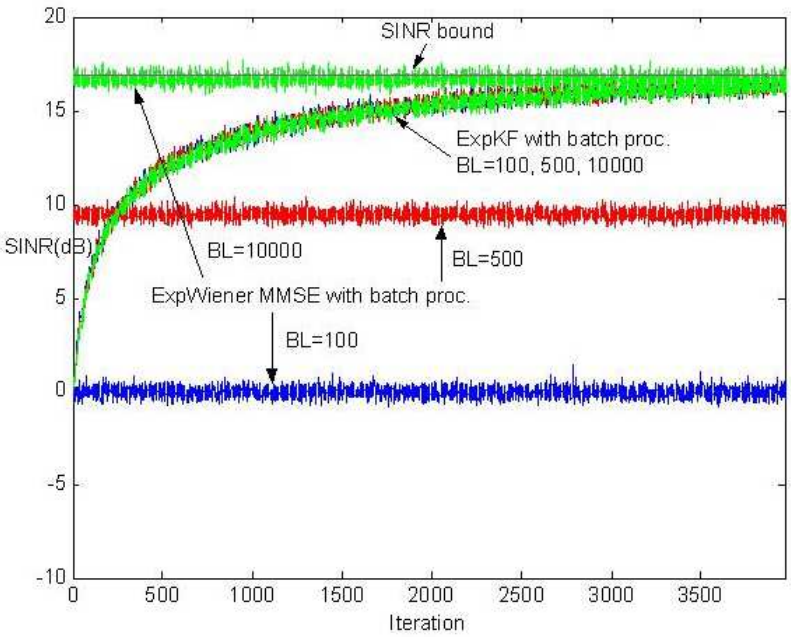

(a) channel spread $=25$.

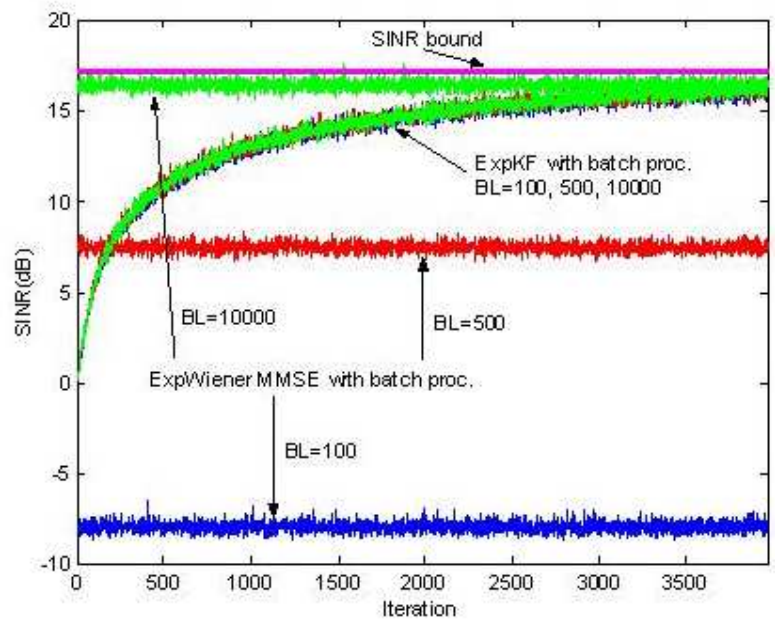

(b) channel spread $=56$.

Fig. 2. $(K=10, N=31, \mathrm{SNR}=20 \mathrm{~dB})$ SINR performance of the expanded subspace Kalman filter-based adaptive detector in multipath channels. Subspace is estimated by windowed PAST algorithm. 